\title{
The Development of Viscometer for On-Site Measuring Lubricating Oil
}

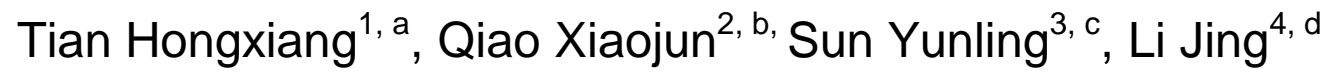 \\ ${ }^{1}$ College of Power Engineering, Naval University of Engineering, Wuhan 430033, P. R. China \\ ${ }^{2}$ The 91439 Unit of PLA, Lvshun 116041, P. R. China \\ ${ }^{3}$ College of Power Engineering, Naval University of Engineering, Wuhan 430033, P. R. China \\ ${ }^{4}$ College of Power Engineering, Naval University of Engineering, Wuhan 430033, P. R. China \\ ahxtianwuhan@aliyun.com, ${ }^{\mathrm{b}}$ 408943102@qq.com, \\ 'Sunyunling315@163.com, 'Leejing0820@163.com
}

Keywords: Hele-Shaw flow, oil monitoring, viscosity, on-site monitoring, sensor.

Abstract. Aim at the problem of on-site measuring mechanical equipment lubricating oil viscosity, the principle of Hele-Shaw flow is chosen to develop viscometer after comparing the characteristics of existing viscosity measuring instruments. Studying on the flowing characteristics of Hele-Shaw flow in the narrow gap, the equation of fluid viscosity with passing through time was deduced according to the law of fluid. The mechanical sensor and signal circuit were designed, and their performances were studied. With compared to the standard method, the results correlation coefficient between a capillary viscometer and the developed device is 0.99 .

\section{Introduction}

For mechanical equipment, the lubrication is essential. The viscosity is one of the most important physical and chemical performance parameters of lubricating oil ${ }^{[1]}$, and is one of the main factors to determine the thickness of lubricating oil film. At present, the commonly methods and equipment used for measuring viscosity of lubricating oil are as follows: capillary viscometers ${ }^{[2]}$, falling ball viscometers $^{[2]}$, rotational viscometers ${ }^{[2]}$, flow on the plate method ${ }^{[3]}$, quartz tuning fork viscometers ${ }^{[4]}$, vibrational viscometers ${ }^{[5]}$, orifice viscometers ${ }^{[6]}$, ultrasonic viscometers ${ }^{[7]}$, et al. These methods have their own features, however, the careful consideration and research are needed to satisfy easily using and accurately measuring on the spot. The viscometer based on the principle of Hele-Shaw flow is a promising field method ${ }^{[8]}$.

\section{The principle of viscometer based on Hele-Shaw flow}

Hele -Shaw flow which is in a very narrow gap between two parallel plates is a kind of special form of Stokes Flow ${ }^{[9]}$.

There are the following assumptions about oil and flow:

the oil to be incompressible, (2)the oil to be Newtonian fluid; (3)the slit to be long enough and uniform in width; (4) oil in the slit wall without sliding; (5) flow being steady (6)flow being laminar.

As shown in figure 1, the oil kinematic viscosity value is proportional to the time $t$, in which the oil flowing through the distance $S$, as shown in formula (1).

$$
v=\frac{g V H^{2}}{2 A S^{2}} \ln \left(1+\frac{S}{L_{0}}\right) \cdot t
$$

where,

$v$ is a kinematic viscosity of the liquid.

$\mathrm{g}$ is a constant of $9.8 \mathrm{~m} / \mathrm{s}^{2}$.

$\mathrm{V}$ is the defined volume of the liquid flowed during tested. 
$\mathrm{H}$ is half of the slit width.

$\mathrm{A}$ is the oil bottom area.

$\mathrm{S}$ is the distance the liquid flowing through.

L0 is initial length which is constant for certain sensor.

$t$ is the time required for this volume of fluid to flow through the length of $S$.

Under the condition of these assumptions mentioned above, the formula (1) shows that oil kinematic viscosity value is proportional to the time $t$ required for this volume of fluid to flow through the length of $S$, namely, the proportion coefficient is

$$
k=\frac{g V H^{2}}{2 A S^{2}} \ln \left(1+\frac{S}{L_{0}}\right)
$$

Each parameters of proportion coefficient $\mathrm{k}$ are concerened with measuring instrument. Proportion coefficient $\mathrm{k}$ can be calculated by the parameters, can also be obtained by the certified viscosity reference.

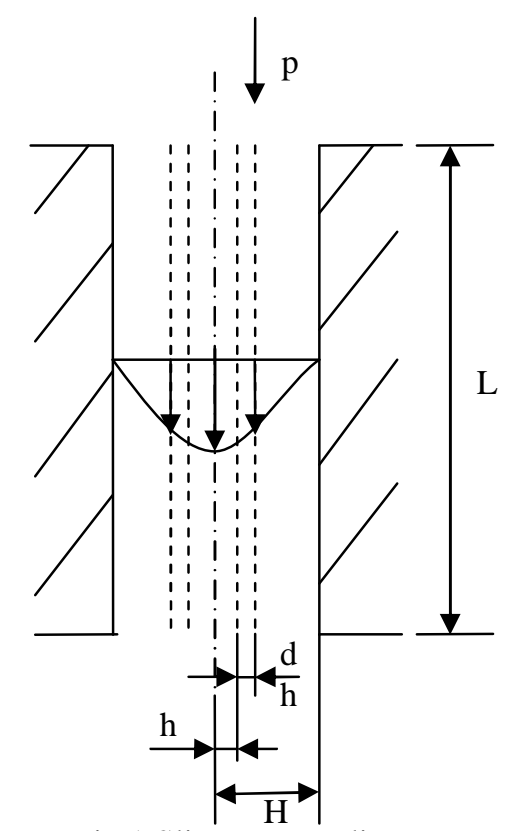

Fig.1 Slit structure diagram

\section{The design of viscometer based on Hele-Shaw flow principle}

The viscometer principle is shown in figure 1, real measuring plate as shown in figure 2.

\section{The design of the measurement circuit}

The measurement circuit consists of transmitting circuit, receiving circuit, amplifying circuit and single-chip microcomputer processing signal, as shown in figure 3 . The measuring results, which are output signal from the receiver and then processed by single-chip microcomputer, are shown on the computer screen. 


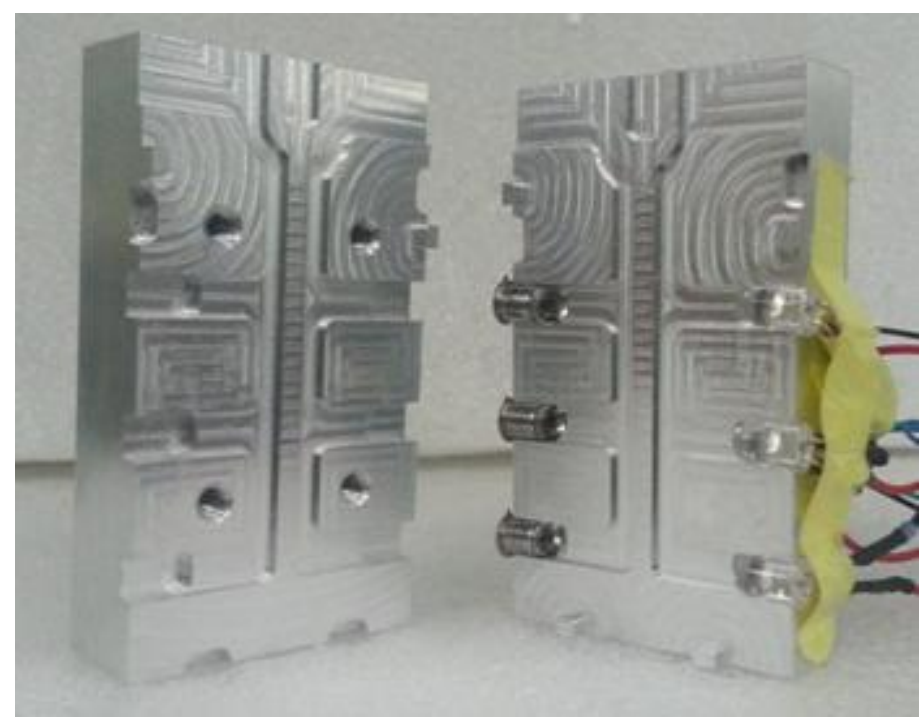

Fig.2 Real measuring plate

infrared light

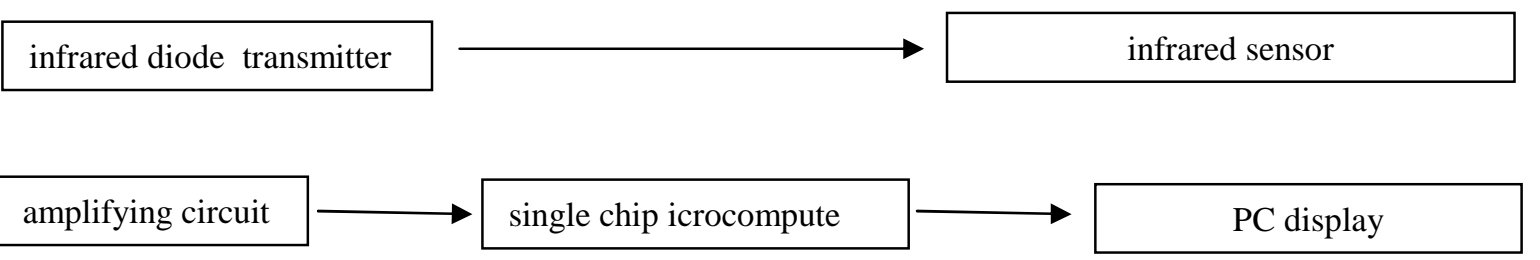

Fig.3 Measurement circuit structure diagram

\section{The measuring performance of the viscometer based Hele-Shaw flow principle}

The 8 oil samples were made up by mixing lubricating oil CD40-15W with fuel at different ratio in laboratory. The viscosity results of 8 oil samples are listed in table 1 at $40{ }^{\circ} \mathrm{C}$ by comparing the standard capillary viscometer with the developed viscometer.

Table1 The results of the standard capillary viscometer and Hele-Shaw viscometer

\begin{tabular}{|c|c|c|c|c|c|c|c|c|c|}
\hline \multicolumn{2}{|c|}{ oil samples } & $1 \#$ & $2 \#$ & $3 \#$ & $4 \#$ & $5 \#$ & $6 \#$ & $7 \#$ & $8 \#$ \\
\hline \multirow{2}{*}{$\begin{array}{l}\text { the standard } \\
\text { capillary } \\
\text { viscometer }\end{array}$} & mean viscosity/cst & 95.07 & 89.80 & 85.43 & 80.73 & 78.27 & 73.33 & 68.97 & 65.84 \\
\hline & standard deviation /cst & 0.40 & 0.42 & 0.31 & 0.25 & 0.26 & 0.27 & 0.19 & 0.16 \\
\hline \multirow{2}{*}{$\begin{array}{l}\text { Hele-Shaw } \\
\text { viscometer }\end{array}$} & mean viscosity/cst & 96.1 & 87.3 & 86.1 & 78.6 & 75.8 & 74.7 & 67.4 & 64.9 \\
\hline & standard deviation /cst & 2.4 & 2.5 & 2.1 & 2.2 & 1.9 & 1.9 & 1.8 & 1.8 \\
\hline
\end{tabular}

The results correlation coefficient between standard capillary viscometer and the developed device is 0.99. From table 1, it is shown that the results standard deviation of Hele-Shaw viscometer is more than of the standard capillary viscometer.

\section{Conclusions}

The experiments demonstrate that the developed viscometer based on Hele-Shaw flow is feasible for measuring the oil viscosity on site. The results correlation coefficient between standard capillary viscometer and the developed device is 0.99 . 


\section{Acknowledgements}

This work was financially supported by the Hubei Province Natural Science Foundation (2010CDB01505).

\section{References}

[1] Xinping Yan: Condition Monitoring and Fault Diagnosis for Mechanical System. Wuhan: Wuhan University of Technology Press, 2009, pp91-92.

[2] GB/T 10247-2008. Methods of Viscosity Measurement. 2009.

[3] Liu Tao, Tian Hongxiang, and Liu Yu: Lubrication Engineering, June 2009, Vol.34, No.6:96-99.

[4] Huang Xianjin: Lubricating Oil Quality Sensor Design Based-on Quartz Tuning Fork. Nanjing University of Science and Technology, 2014.03.

[5] Keiichi Higashino, Hiroshi Yabuno, Kazuhiko Aono, et al: Journal of Vibration and Acoustics, DECEMBER 2015, Vol. 137. DOI: 10.1115/1.4030975.

http://vibrationacoustics.asmedigitalcollection.asme.org/ on 12/26/2015

[6] Javier Rondona, Maria A. Barrufet, Gioia Falcone, et al: Flow Measurement and Instrumentation, 23 (2012) 9-18.

[7] Wunderlich T,Brunn P O: Flow Measurement and Instrumentation ,1999,10(4): 201-205.

[8] Qiao Xiaojun: The Study of Viscosity On-Site Measurement for Marine Lubricant. Wuhan: The Naval University of Engineering, 2013.10.

[9 Milne L M: Theoretical hydrodynamics. New York: Courier Dover Publications,1996: 144-150. 